\title{
Probing the nuclear medium by antiprotons
}

\author{
Horst Lenske \\ Institut für Theoretische Physik, Universität Giessen, \\ Heinrich-Buff-Ring 16, D-35392 Giessen, Germany
}

\begin{abstract}
We propose to use anti-protons to investigate the sizes of stable and neutronrich exotic nuclei by measurements of the $\bar{p} A$ absorption cross section along isotopic chains in inverse kinematics. The expected effects are studied theoretically in a microscopic model. The $\bar{p} U$ optical potentials are obtained by folding free space $\bar{p} N$ scattering amplitudes with HFB ground state densities and solving the scattering equations by direct integration. The mass dependence of the absorption cross sections and of the nuclear radii are found to be directly related by a scaling law.
\end{abstract}

\section{Introduction}

A highly interesting question of modern nuclear structure physics is to understand the evolution of nuclear shapes and sizes from stable to $\beta$ - unstable nuclei. Experimentally, a variety of efforts is undertaken or in planning to investigate properties of neutron-rich exotic nuclei $[1,2,3]$. Here, we investigate anti-protons as probes for nuclear sizes of exotic neutron-rich nuclei. A more detailed discussion is found in [4]. Hitherto, e.g. at BNL $[5,6]$ or LEAR $[7,8,9,10]$, the experiments have been performed with secondary anti-proton beams on stable nuclei in fixed target geometry and standard kinematics, actually using stopped anti-protons. Obviously, such experimental configurations are not applicable for reactions on short-lived isotopes. Since they are available also only as secondary beams, the solution are measurements in colliding beam geometry, proposed recently in [11] and becoming feasible at the new FAIR facility at GSI [13]. The FAIR plans include as a central component $\bar{p}$ production and accumulation facilities. For nuclear structure research the New Experimental Storage Ring (NESR) for short-lived nuclides and an intersecting electron accelerator for colliding beam experiments will be available [12]. With moderate modifications on an acceptable level the NESR/ $e^{-}$setup can be converted into a $\bar{p} A$ collider facility [11]. 
The physics of $\bar{p} A$ interactions is by itself an interesting topic for hadron physics brought to the attention of nuclear physics originally by Duerr and Teller [14]. The usefulness of $\bar{p}+A$ scattering for studies of the neutron skin in stable medium and heavy nuclei was realized some years later in an experiment at BNL [5]. Since then, a variety of measurements have been performed, e.g. [7, 8, 9, 10]. As for the elementary anti-proton nucleon $(\bar{p} N)$ vertex the $\bar{p} A$ reactions are dominated by processes in which the incoming anti-proton annihilates on a target nucleon into a variety of particles producing typically and preferentially a shower of pions as the final result. Here, we utilize the strong $\bar{p} A$ absorption for nuclear structure investigations without paying particular attention to the hadron physics aspects, some of which, however, can - and in another context will - also be studied with the facility under discussion.

The very details of $\bar{p} N$ annihilation in a nuclear medium are not of our interest in the present context. For our purpose we are satisfied to know that the elementary annihilation processes result finally in a strong suppression of the incoming flux. In the elementary elastic scattering amplitude $f_{\bar{p} N}$ the annihilation channels are observed as a strong imaginary part and a small, almost vanishing, real part. Correspondingly, in inclusive $\bar{p} A$ elastic scattering the annihilation channels can be accounted for globally by a strongly absorptive optical potential, as e.g. in [17, 18, 19, 20]. Very often, the real parts are set to zero or are taken to be very small compared to the imaginary parts.

The theoretical approach is described in sect. 2. As a typical example results for the $N i$ isotopic chain are discussed in sect.3. We find a direct proportionality between the mass dependence of the absorption cross sections and the target root mean square radii.

\section{Microscopic description of $\bar{p} A$ interactions}

\subsection{The $\bar{p} A$ optical potential and elastic scattering}

The coordinate space $\bar{p} A$ optical potential at energy $T_{L a b}$ is obtained in the impulse or $t \rho$ approximation,

$$
U_{\text {opt }}(\mathbf{r})=\sum_{N=p, n} \int \frac{d^{3} q}{(2 \pi)^{3}} \rho_{N}(q) t_{\bar{p} N}\left(T_{L a b}, q^{2}\right) e^{i \mathbf{q} \cdot \mathbf{r}},
$$

given in terms of the elementary $\bar{p} N$ momentum space T-matrix $t_{\bar{p} N}$ and the Fourier transforms $\rho_{p, n}(q)$ of the proton and neutron ground state densities. The result of the folding calculation is a complex optical potential $U_{\text {opt }}=U_{c}+V+i W$ with real and imaginary parts $V$ and $W$, respectively, and to be supplemented by the Coulomb potential, obtained from the HFB charge densities. We also find $U_{\text {opt }}=U_{o p t}^{(p)}+U_{o p t}^{(n)}$. As in free $\bar{p} N$ scattering the potentials are dominated by the strong imaginary parts. Overall, the radial shapes of the folding potentials resemble closely Wood-Saxon form factors.

We use a non-relativistic approach and describe the $\bar{p} A$ scattering by solving the optical model Schroedinger equation

$$
\left(-\frac{\hbar^{2}}{2 \widetilde{m}} \vec{\nabla}^{2}+U_{\text {opt }}-T_{\text {lab }}\right) \Psi^{(+)}(\mathbf{k}, \mathbf{r})=0
$$


Here, $\Psi^{(+)}(\mathbf{k}, \mathbf{r})$ is the optical model wave with asymptotically outgoing spherical wave boundary condition, describing the antiproton-nucleus relative motion in the center-ofmass system with reduced mass $\widetilde{m}$. Eq.2 is solved in partial wave representation and from the solutions we find the partial wave S-matrix elements $S_{\ell j}[26]$,

$$
S_{\ell j}=\eta_{\ell j} e^{2 i \delta_{\ell j}}
$$

given in terms of the inelasticity $\eta_{\ell j} \leq 1$ and the scattering phase shift $\delta_{\ell j}$, respectively, which both are real quantities. The partial wave absorption cross sections are then found as

$$
\sigma_{a b s}(\ell j)=\frac{\pi}{k^{2}} \frac{2 j+1}{2 s+1}\left(1-\left|S_{\ell j}\right|^{2}\right)=\frac{\pi}{k^{2}} \frac{2 j+1}{2 s+1}\left(1-\eta_{\ell j}^{2}\right),
$$

where $s=\frac{1}{2}$ is the spin of the incoming $\bar{p}$. The total absorption cross section is obtained by summing over the partial wave contributions.

An interesting relation is obtained from the quantum mechanical continuity equation

$$
\sigma_{a b s}=\sum_{\ell j} \sigma_{a b s}(\ell j)=-\frac{2 \widetilde{m}}{k \hbar^{2}} \frac{1}{2 s+1} t_{s}\left(\int d^{3} r \Psi^{(+) \dagger}(\mathbf{k}, \mathbf{r}) W\left(\mathbf{r} \Psi^{(+)}(\mathbf{k}, \mathbf{r})\right),\right.
$$

expressing $\sigma_{a b s}$ directly in terms of the absorptive potential $W(\mathbf{r})=\Im U_{\text {opt }}(\mathbf{r})$. Since $W=W_{p}+W_{n}$ we conclude immediately that also $\sigma_{a b s}=\sigma_{a b s}^{(p)}+\sigma_{a b s}^{(n)}$ is additive in the proton and neutron partial contributions, describing the annihilation of the incoming $\bar{p}$ on the target protons and neutrons, respectively.

\section{$3 \bar{p}$ Annihilation on $\mathrm{Ni}$-isotopes}

\subsection{Antiproton absorption and nuclear radii}

HFB results, obtained by the DME approach [27], for rms-radii of the proton and neutron ground state densities are displayed in Fig.1, respectively. Also, we find a very satisfactory overall agreement of the theoretical and the measured binding energies on the level of $5 \%$.

The total absorption cross sections for Ni-Isotopes in the mass range $48 \leq \mathrm{A} \leq 88$ for a variety of antiproton incident energies between $\mathrm{T}_{\text {Lab }}=50 \mathrm{MeV}$ up to $\mathrm{T}_{\text {Lab }}=400 \mathrm{MeV}$ are shown in Fig.2. We first consider the mass dependence of $\sigma_{a b s}$. As a common feature at all energies we notice the continuous increase with mass number, i.e. with the neutron access. By comparison to Fig.1 a striking similarity between the slopes of $\sigma_{a b s}$ and the rms-radii is detected.

In order to understand the mechanism behind this correlation the absorption cross sections at $\mathrm{T}_{L a b}=200 \mathrm{MeV}$ and $\mathrm{T}_{L a b}=400 \mathrm{MeV}$ are compared directly to the nuclear rms-radii in Fig.3. From Fig.3 we realize that the slope of $\sigma_{a b s}$ follows closely the mass dependence of the rms radii. We find this behavior at all energies considered here in an almost universal manner. In order to emphasize this close relationship, the root-meansquare $(\mathrm{rms})$ radii of the nuclear mass distributions from Fig.1 were added to the figure by normalizing the rms-radii to the cross sections at ${ }^{58} \mathrm{Ni}$ for both energies. Hence, we conclude that antiproton annihilation is an appropriate probe for nuclear sizes. 


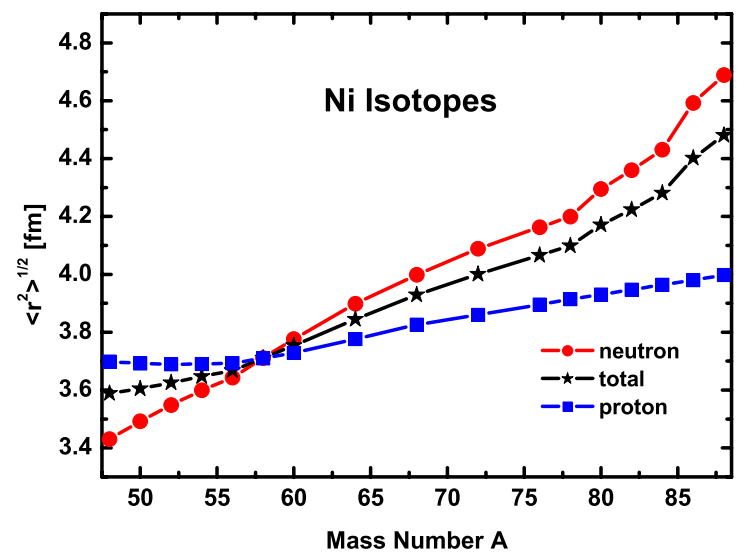

Figure 1: Root-mean-square (rms) radii for ${ }^{48-88} \mathrm{Ni}$-isotopes. HFB results for protons (squares), neutrons (circles) and the total (isoscalar) density (stars) are shown. The mass range corresponds to the isotopes found in the HFB calculations to be particle-stable. The lines are to guide the eye. Note the change from proton skins at $A \lesssim 58$ to neutron skins at larger mass numbers.

The partial absorption cross sections due to separate annihilation on the target protons and neutrons, respectively, are displayed in Fig.3. Again, the mass dependence of the partial proton and neutron absorption cross sections is remarkably close to the rms-radii of the corresponding nuclear density distributions, as seen by comparing to Fig.1.

The energy dependence of $\sigma_{a b s}$ reveals an interesting saturation effect which apparently has not been reported before. With increasing energy the magnitude of $\sigma_{a b s}$ decreases but close to $T_{l a b}=400 \mathrm{MeV}$ a kind of converging behavior set in, appearing as a slow approach towards an asymptotic value. The reason behind this saturation-like energy dependence is found in Fig.4. There, the quantum mechanical partial wave cross sections, eq.4, averaged over the total angular momentum $j=\ell \pm \frac{1}{2}$, are shown as functions of the impact parameter $b_{\ell}$, where $b_{\ell} k=\eta_{c}+\sqrt{\eta_{c}^{2}+\ell(\ell+1)}$ with $\eta_{c}$ the Sommerfeld parameter. Although $b_{\ell}$ is a semi-classical quantity it is useful for understanding the relation of the absorption process to the target geometry. The results of Fig. 4 show that the antiproton absorption process is indeed dominated by annihilation in the nuclear tail and surface regions. However, with increasing incident energy the absorption maximum moves into the surface region until the strong absorption in the central region is encountered. Once the energy is such that this condition is reached $\sigma_{a b s}$ approaches an asymptotic value 


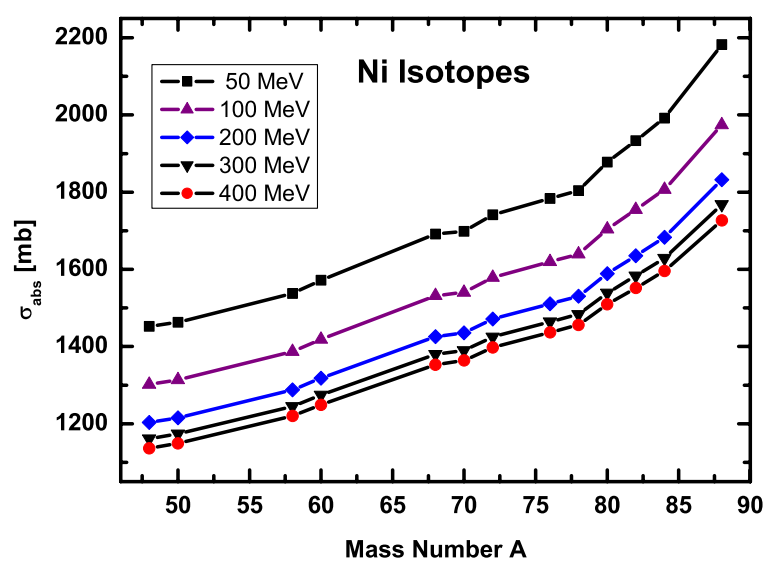

Figure 2: Absorption cross sections for antiproton annihilation on the ${ }^{48-88} \mathrm{Ni}$ isotopes at various antiproton incident energies.

constrained by the black disk limit $\sigma_{a b s} \sim \pi R_{A}^{2}$ where $R_{A}$ is of the order of the nuclear radius.

This behavior is complementary to the observations made in antiprotonic atoms and in low-energy annihilation on nuclear targets. There, the absorption occurs at in the distant tail region of the nuclear density $[24,25]$ while at the much higher energies considered here the annihilation occurs closer to the central region of the target nucleus.

\subsection{Screening in neutron-rich nuclei}

Further insight into the reaction mechanism of $\bar{p} A$ interactions is gained by inspection of the absorption along a trajectory at fixed impact parameter. For that purpose we use the eikonal approximation [26] in which the cross section for absorption on the target nucleons $q=p, n$ at a given impact parameter $b$ can be expressed as

$$
\sigma_{a b s}^{(q)}(b)=2 \pi \int_{-\infty}^{+\infty} d z \Pi_{q}(z, b)
$$

In eikonal approximation the absorption kernel $\Pi_{q}(z, b)$ can be evaluated further and we find the expression

$$
\Pi_{q}(z, b)=\frac{1}{2 s+1} \operatorname{tr}_{s}\left\{\Pi^{(s)}(z, b)\right\}=-\frac{2 \widetilde{m}}{k \hbar^{2}} e^{-2 \chi_{q}(z, b)} W_{q}(z, b) \quad,
$$




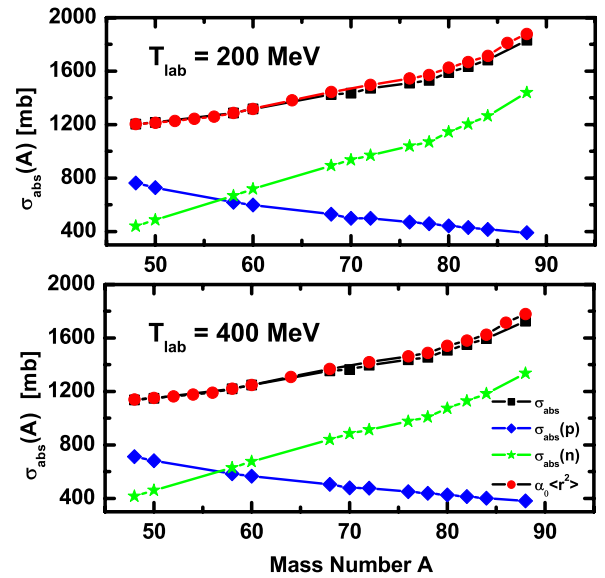

Figure 3: Absorption cross sections (circles) for antiproton annihilation on Ni-isotopes at $T_{l a b}=200 \mathrm{MeV}$ and $T_{l a b}=400 \mathrm{MeV}$, respectively, are compared to the rms-radii of the nuclear matter densities (squares). In both cases, the rmsradii are arbitrarily normalized to $\sigma_{a b s}$ for ${ }^{58} \mathrm{Ni}$ by multiplication with an energy dependent constant $\alpha_{0}=\alpha_{0}\left(T_{l a b}\right)$. Also shown are the partial cross sections for absorption on the target neutrons and protons.

including the trace over the spin projection by assuming a spin-independent $W_{q}$. The absorption of the incoming antiproton flux along the trajectory through the target nucleus is described by the profile function [26]

$$
\chi_{q}(z, b)=\int_{-\infty}^{z} d \xi \Im K_{q}(\xi, b) \sim-\frac{\widetilde{m}}{k \hbar^{2}} \int_{-\infty}^{z} d \xi W_{q}(\xi, b) .
$$

where $K_{q}(z, b)$ is the local wave number, including the potential. The value of $\Pi(z, b)$ is a measure for the degree of absorption at a given point in the scattering plane, defined in coordinate space by $(z, b)$. The case $\Pi(z, b)=0$ indicates full transmission, e.g. as it naturally occurs outside the $\bar{p} A$ interaction zone. Overall, for impact parameters $b \ll R_{A}$, $R_{A}$ the target radius, the incoming flux is strongly absorbed, assigning the characteristics of a black disk to the target. Hence, configurations with $b \gtrsim R_{A}$ are of greater physical interest because they are favorable for the intended measurements.

Assuming for $W(r)$ a gaussian radial form factor we find the analytic result $\sigma_{a b s}\left(A, T_{c m}\right)=$ $\pi<r^{2}>_{A} f\left(A, T_{c m}\right)$ and the scaling function $f\left(A, T_{c m}\right)$ can be calculated in closed form where $f\left(A, T_{c m}\right)$ is given by the dynamics of the $\bar{p} A$ system [4]. Indeed, approximating $W(r)$ by a Gaussian of the same volume integral the two independently derived quantities are numerically surprisingly close, e.g. $\alpha_{0} / \alpha \simeq 1.02$ at $T_{L a b}=400 \mathrm{MeV}$. The eikonal 


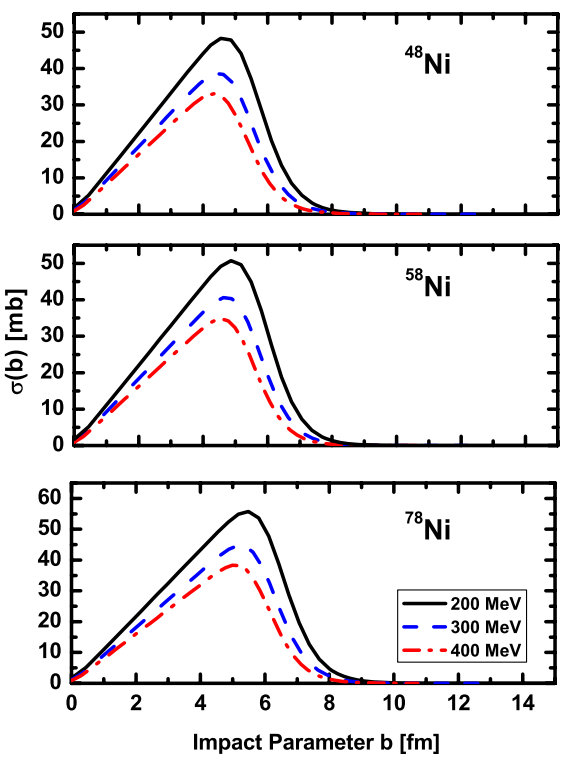

Figure 4: Partial wave absorption cross sections for antiproton absorption on the ground state density distributions of Ni-isotopes at various incident energies. The quantum mechanical results are shown as functions of the impact parameter $\mathrm{b}=\mathrm{b}_{\ell}$.

scaling factor also explains the apparent convergence of the absorption cross sections to limiting asymptotic values as indicated in Fig.2.

Results for $\Pi(z, b)$ at an incident $\bar{p}$ energy of $T_{l a b}=300 \mathrm{MeV}$ are displayed in Fig. 5 for a variety of $N i$ nuclei. The impact parameter is chosen to be $b=4 \mathrm{fm}$ which roughly fixes the scattering to grazing collisions over the mass region considered in Fig.5. We find an interesting evolution with the neutron number: In ${ }^{48} \mathrm{Ni}$ the absorption occurs mainly on the protons which in that neutron-deficient nucleus are sticking out, as seen from the rms values in Fig.1. In the stable $A=58$ isotope with almost equal number of protons and neutrons and negligible excess density the antiprotons are equally well absorbed on both neutrons and protons. By further adding neutrons the absorption on the neutron excess density obviously is strongly increasing, overshooting the absorption on protons by a factor of about 2 towards ${ }^{78} \mathrm{Ni}$. Hence, the neutron-rich nuclei the skin density shields the target protons by absorbing the incoming antiprotons before they reach the region of the much stronger bound and therefore spatially much stronger confined protons. Hence, we find an intriguing interplay between neutron skins and $\bar{p}$ annihilation which is causing the decrease of the partial proton cross sections seen in Fig.3. 


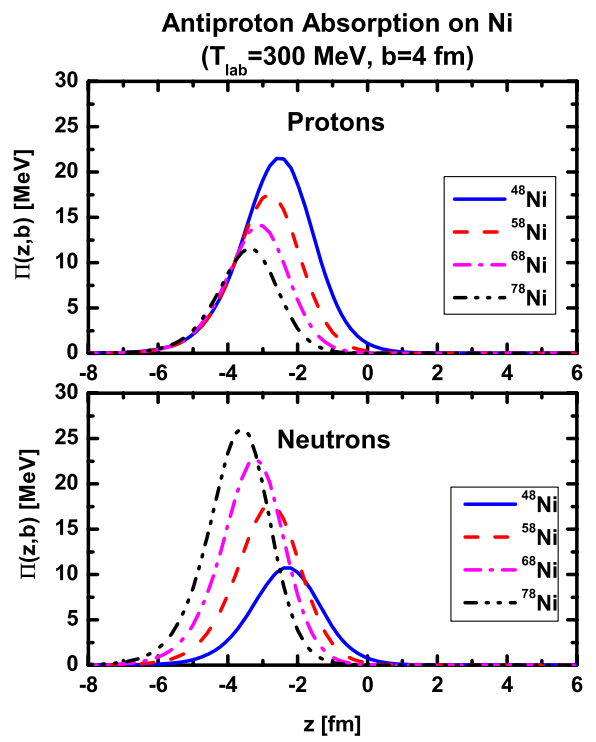

Figure 5: Absorption of the incoming antiproton along a trajectory at an impact parameter $b=4 \mathrm{fm}$. The absorption kernel, eq.7, is displayed for annihilation of antiprotons with an incident energy of $T_{l a b}=300 \mathrm{MeV}$ on ${ }^{48,58,68,78} \mathrm{Ni}$. The $\bar{p}$ beam is incoming from the left, i.e. from $z=-\infty$. Note the reversed behavior of the $\bar{p}$ absorption on protons and neutrons with increasing neutron excess.

This remarkable selectivity opens promising perspectives in at least two directions, namely

- it clearly shows that antiprotons are a probe well suited for investigations of neutron (and proton) skins;

- it indicates a new method for gaining information on antiproton-neutron interactions on which the data base is very scarce.

The latter point is of particular interest because it adds a new aspect to the experiment proposed in [11]: Scanning over an isotopic chain will allow to record the gradual change from a $\bar{p} p$ to $\bar{p} n$ annihilation, hence providing a new access to the pbar-nucleon interactions. 


\section{Conclusions and outlook}

Antiproton-nucleus scattering was investigated theoretically as a tool for measuring the sizes of neutron skins in exotic nuclei. Deriving the $\bar{p} A$ optical potentials microscopically in a folding approach from the elementary free space $\bar{p} N$ scattering amplitudes and HFB density distributions we found a selectivity of the annihilation process along the trajectory of the anti-proton in the nuclear medium on the proton and neutron density distributions. Our results show that simple absorption cross section measurements by comparing beam currents before and after the target zone will already provide valuable new information on the densities of the target nuclei, most likely of a higher accuracy than achievable by other methods. More involved measurements seem to be feasible from which more detailed information on the configurations of the target nuclei can be obtained.

A next step will be to consider less inclusive experiments. An appropriate approach is to gate on the $A-1$ nucleus at fixed $Z$, corresponding to measurements of the annihilation of the incoming $\bar{p}$ on a single target neutron. Such data are of interest for nuclear structure physics because of their selectivity on annihilation on a valence particle. However, they are also of interest for hadron physics by providing information on antiproton-neutron interactions.

Parallel to the nuclear structure studies the experimental program should also envision investigations of elastic $\bar{p} A$ interactions. A consistent and much extended set of elastic angular distributions is highly desired by theory, helping to understand the antiproton self-energies in nuclear matter.

Acknowledgement:

Supported in part by DFG, contract Le 439/05, and BMBF.

\section{References}

[1] D. Cortina-Gil et al., Nucl.Phys. A718 (2003) 431; P. Egelhof, Nucl. Phys. A22 (2003) C254; G. D. Alkhazov et al., Nucl.Phys. A712 (2002) 269.

[2] A. Krasznahorkay et al., Phys. Rev. Lett. 82 (1999) 3216; A. Krasznahorkay et al., Proc. of the INPC 2001, Berkeley, Aug. 2001, ed. by E. Norman et al., p.751 (2001).

[3] H. Lenske, G. Schrieder, Eur.Phys.J. A2 (1998) 41; H. Jeppsen, H. Lenske, G. Schrieder et al., Phys.Lett. B (2005) (submitted).

[4] H. Lenske, P. Kienle, Phys. Lett. B (2005) in print, e-print: nucl-th/0502065.

[5] W. M. Bugg, G. T. Condo, E. L. Hart, H. O. Cohn, R. D. McCulloch, Phys. Rev. Lett. 31475 (1973).

[6] V. Ashford, M. E. Sainio, M. Sakitt, J. Skelly R. Debbe, W. Fickinger, R. Marino, and D. K. Robinson, Phys. Rev. C 301080 (1984)

[7] S. Janouin et al., Nucl.Phys. A451 (1986) 541.

[8] G. Bruge et al., Phys. Lett. B169 (1986) 14.

[9] P. Lubinski et al., Phys. Rev. Lett. 73 (1994) 3199.

[10] S. Wycech, J. Skalski, R. Smolanczuk, J. Dobaczewski, J.R. Rook, Phys. Rev. C54 (1996) 1832. 
[11] P.Kienle, NIMB 214 (2004) 193.

[12] I.A. Koop et al., Proc. EPAC 2002, Paris, p. 620.

[13] Proposal for the GSI Future Project, http://www.gsi.de.

[14] H.-P. Duerr, E. Teller, Phys. Rev. 101494 (1956).

[15] Carlo Guaraldo, Nuovo Cim. A102 (1989) 1137.

[16] J.A. Niskanen, Anthony M. Green, Nucl.Phys. A404 (1983) 495.

[17] T. Suzuki, Nucl.Phys. A444 (1985) 659.

[18] H. Heiselberg, A.S. Jensen, A. Miranda, G.C. Oades, J.M. Richard, Nucl.Phys. A451 (1986) 562 .

[19] S. Adachi, H.V. Von Geramb, Nucl.Phys. A470 (1987) 461.

[20] Zhang Yu-shun, Liu Ji-feng, B. A. Robson, and Li Yang-guo, Phys.Rev. C54 (1996) 332.

[21] A.M. Green, J.A. Niskanen, Quarks and Nuclei, ed. W. Weise, World Scientific, Singapore 1984.

[22] Tan Zhen-Qiangi and Gu Yun-Ting, J. Phys. G: Nucl. Part. Phys 15 (1989) 1699.

[23] G. Faldt and I. Hultage, J. Phys. G: Nucl. Phys. 4 (1978) 363.

[24] C.J. Batty, E. Friedman, A. Gal, Phys. Rep. 287 (1997) 385.

[25] A. Trzcinska, J. Jastrzebski, and P. Lubinski, F. J. Hartmann, R. Schmidt, and T. von Egidy, B. Klos, Phys.Rev.Lett. 87 (2001) 082501-1.

[26] Charles J. Joachain, Quantum Collision Theory, North-Holland, 1984.

[27] F. Hofmann, H. Lenske, Phys. Rev. C57 (1998) 2281.

[28] H. Lenske, F. Hofmann, C.M. Keil, Rep.Prog.Nucl.Part.Phys. 46 (2002) 187.

[29] G. Audi and A. H. Wapstra, Nucl. Phys. A595 409 (1995). 